\title{
MANEUVER-BASED CROSS-VALIDATION APPROACH FOR ANGLE-OF-ATTACK ESTIMATION
}

\author{
Alberto Brandl ${ }^{1, *}$, Manuela Battipede ${ }^{2}$ \\ ${ }^{1}$ Polytechnic University of Turin, c.so Duca degli Abruzzi, 24 - Turin (Italy), alberto.brandl@ polito.it \\ ${ }^{2}$ Polytechnic University of Turin, c.so Duca degli Abruzzi, 24 - Turin (Italy), \\ manuela.battipede@polito.it
}

Key words: AOA Estimation, Cross-Validation, Neural Network, Machine Learning, Synthetic Sensor

\begin{abstract}
The estimation of the Angle of Attack (AOA) and Angle of Sideslip (AOS) is crucial for flight monitoring and control. However, a gap has been identified on the data selection technique for the class of estimators based on data-driven methods, such as the synthetic sensor based on Neural Network (NN). This paper proposes a Cross Validation (CV) technique applied on a manoeuver-based partitioning method to provide evidence that a given selection of data can lead to better estimation performance, with the final aim of providing a list of manoeuvers suitable for the training phase of the estimator. Results are shown using simulated data related to the CleanSky 2 project MIDAS.
\end{abstract}

\section{INTRODUCTION}

In 2019, two tragedies related to the failure of the Air Data System (ADS) led to a growing interest in synthetic sensors for aerodynamic angles. Despite the several solutions available in literature, only few of them actually reached Technology Readiness Level (TRL) higher than 6 . The reasons behind the missing technological transfer might be found on the difficulty of the aeronautical community of comparing the performance of the Commercial Off-The-Shelf (COTS) probes with the performance obtained with a synthetic estimator. The term aerodynamic angles usually refers to the angles between the velocity vector and the Body axis of an aerial vehicle. These two angles, Angle of Attack (AOA) and Angle of Sideslip (AOS), are related to the generation of forces and moments on the aircraft and this makes them very important for a safe flight. The typical equipment used to measure these two angles is a certain number of angle vanes or multihole probes that are mounted protruding the fuselage of the aircraft [1]. On the contrary, in this field a synthetic sensor (also called virtual sensor) is an estimator able to provide AOA and/or AOS with an sufficiently high accuracy without the support of dedicated protruding probes, although it might use other protruding probes such as a Pitot probe for the measurement of the total pressure. In the past, researchers applied several techniques to obtain a synthetic sensor for AOA and AOS, for instance several studies exist on the application of Kalman Filter (KF) [2-8], other studies focus on the application of Neural Network (NN) [9-11], while the mathematical description of the problem resulting in an analytical estimator independent from the aircraft have been published in [12]. Another important research topic is the wind estimation, which is tighly related to the estimation of AOA/AOS [13].

The MIDAS project is funded by Clean Sky 2 with the aim of design and manufacture a modular, fully 
integrated and digital ADS equipped with synthetic sensors based on NN [14-17]. Started at the end of 2018, it is following a certifiable process based on DO-254 and DO-160 and in 2021 it will constitute a rare case of certifiable probe equipped with synthetic estimators of AOA/AOS. Figure 1 shows an high level schematic of the project.

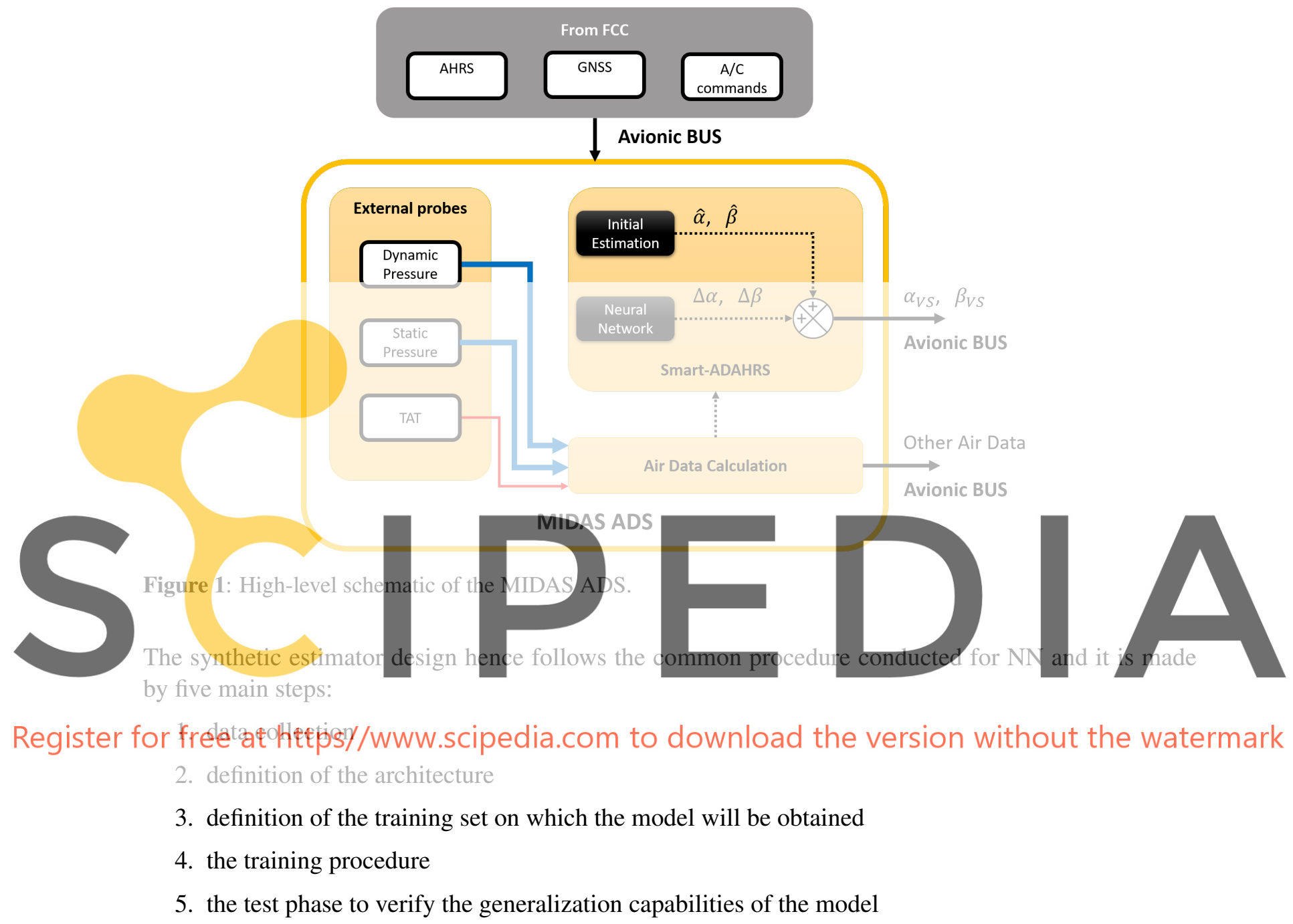

In literature, it is usually studied the choice of the architecture of the $\mathrm{NN}$ and it is typically conducted a comparison of the performance obtained varying some hyper-parameter of the NN, such as the number of neurons. In this design flow, the selection of the training set is still manually conducted following a trial-and-error procedure. Some observations are available from previous works $[18,19]$ but the selection of the data for the estimation of the aerodynamic angles is still an open question.

This work proposes a technique to improve the performance of the data-driven sensor, independently from the architecture. Instead of partitioning by experience the dataset in a training set and a test set, this work proposes to apply a partitioning based on the flight condition, followed by the Cross Validation (CV) technique. It is hence possible to generate several different pairs of training sets and test sets 
and then choose the best estimator that originate from the various possibilities [20]. Moreover, in this work, it is taken in consideration the nature of the available data, which comes from flight data recording or simulation. In this framework, it is possible to define a rule to subdivide each flight so that each partition can be identified with a flight manoeuver. This training procedure has been called Manoeuverbased Cross-Validation (MBCV). Once the MBCV training is conducted, the analysis of the selected training and test sets can help to analyze which are the most suitable manoeuvers and which ones can be discarded. The comparison can be conducted both on the error timeseries and on the uncertainty charts. This paper is composed as follows: Sec. 2 describes the method in details, Sec. 3 shows some preliminary results obtained with the MBCV method. Conclusions are given in Sec. 4.

\section{METHOD}

Once the relevance of a synthetic sensor for AOA/AOS estimation has been assessed, this section contains a description of the method proposed to design an effective synthetic sensor. The aerodynamic angles AOA (or $\alpha$ ) and AOS (or $\beta$ ) are written respectively as the sum of an initial estimation $\hat{\alpha}$ and $\hat{\beta}$ and of a gap $\Delta \alpha$ and $\Delta \beta$, as in Eq. 1
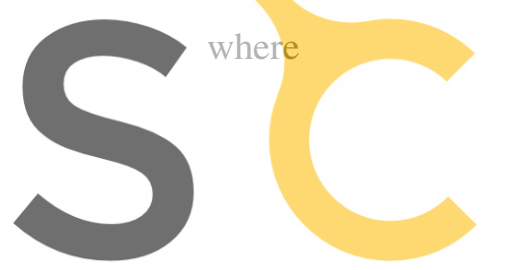
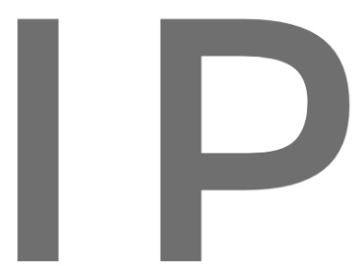

$$
\begin{aligned}
& \alpha_{V S}=\hat{\alpha}+\Delta \alpha \\
& \beta_{V S}=\hat{\beta}+\Delta \beta
\end{aligned}
$$

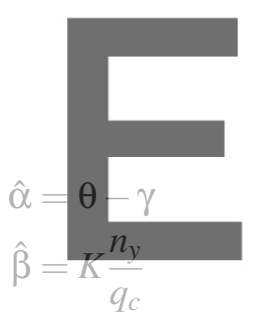

(1)

(2)
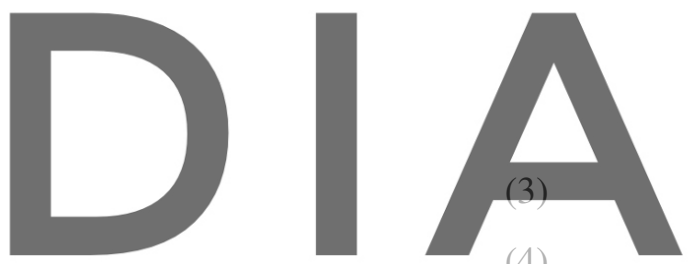

(4)

Register for free at https//www.scipedia.com to download the version without the watermark where $\theta$ is the pitch angle, $\gamma$ is the flight path angle, $n_{i}$ represents a proper acceleration along the $i$ axis and $q_{c}$ is the impact pressure. $K$ is a constant which depends on the application. For what concern $\Delta \alpha$ and $\Delta \beta$, the architecture of the MIDAS synthetic sensors is based on Multilayer Perceptron (MLP), in particular it is a feed-forward fully connected $\mathrm{NN}$ with 16 inputs, 2 hidden layers with 24 neurons each and 2 outputs implementing Eq. 5.

$$
[\Delta \alpha, \Delta \beta]^{T}=\mathbf{f}\left(T A S, \hat{\alpha}, n_{x}, n_{y}, n_{z}, \theta, \varphi, p, q, r, \delta_{e}, \delta_{a}, \delta_{r}, \delta_{t h}, \Delta_{t h}, \delta_{h s}\right)
$$

where TAS is the true airspeed, $\psi$ and $\phi$ are the yaw and roll angles, $p, q, r$ are the body angular rates, $\hat{\alpha}$ is the initial estimation for the AOA.

Several topologies have been analysed during this project, with a different number of neurons and layers, however this paper focuses on the architecture previously mentioned. This should not be seen as a limitation, because once a sufficient capacity of the NN is provided, the conclusions of this work should remain the same. The analysis of the proposed method with different hyper-parameters is hence not 
conducted here and it will be object of future research. This kind of estimator is deeply affected by the data used for the design. To better describe the dependency of the estimator performance on the data, some insight on the NN must be provided. An MLP is a repeated biased linear composition of the output of the preceding neurons and the weights of these compositions are obtained during the training phase in order to fit a subset of data. The asymptotic solution of the training problem results in the multivariate regression of the training dataset. It is hence clear that the selection of the data has a great importance in the design of the virtual sensor. For instance, if the function obtained with the training phase does not cover a region of the flight envelope, there is little probability that the MLP will be able to predict the output in that region.

The training phase is generally conducted with minimization of a cost function, e.g. the sum-of-squares error, starting from a random initial condition for the weights. This nonconvex optimization generally leads to different functions due to local minima in the cost function. To avoid this problem, the training phase has been repeated several times to pick the best model. Moreover, as common in regression analysis, only a subset of the training set is actually used to evaluate gradients and to modify the weights of the NN. The complementary set is used to avoid overfitting during the training phase. However, it can be observed that a training set can be associated with a model if the training procedure is conducted properly and this observation leads the authors to select the final estimator based on the training dataset. The problem of model selection is well-known literature and one of the most common method to face this problem is the CV method [20].

This paper proposes to conduct a CV procedure on a partitioning of the dataset based on the flight manoeuvers, focusing on the estimation of AOA. In fact, it is possible to take advantage of the fact
that data comes from flight recordings or simulation and the partitions can be drectly associated to a
particular flight condition or manoeuver. Moreover, the time history of each flight contains a sequence
of steady-state condition followed by a manoeuver, as shown in Figure 2. Steady Steady Steady

Register for free at https dfiwww.scipedia.com to download the version without the watermark Time

Figure 2: Time histories splitting strategy

Once a NN is trained using a possible subset of the dataset, every obtained model will differ in which manoeuvers have been used for training and, hence, some observation can be drawn. Although still to be demonstrated rigorously, the composition of timeseries to build several training sets seems not to violate the assumptions on which the $\mathrm{CV}$ is based. In fact, the various timeseries are generated from the same function, which is the mathematical model of the aircraft. However, the length of a manoeuver is not standardized and the size of the training set, especially with respect to the size of the test set, is difficult to be kept under control.

The procedure adopted in this paper starts from the definition of a requirement document to the project leader Piaggio Aerospace which provided the consortium with a set of simulated manoeuvers. This flight database contains steady-state flight conditions at different AOA, dynamic conditions such as phugoid, 
dutch-roll, typical identification manoeuvers as the 3-2-1-1 on pitch angle or on the bank angle. To allow the NN to fit highly nonlinear flight conditions, several stall manoeuvers have been also added to the flight database. Each flight has been analysed with the Data-Driven Identification and Generation of Quasi-Steady States (DIGS) algorithm proposed in [18] to mine the quasi-steady state condition. Then a subset of the dataset has been identified as a candidate family of training sets and then partitioned. The partitions are then grouped following the V-Fold CV procedure, to maintain a ratio between the size of the test set and the size of the training set higher than the one obtained with k-Fold CV. In fact, the k-Fold $\mathrm{CV}$ generally results in a training set which covers the $90 \%$ or more of the original dataset, whereas in this paper the minimum ratio between the size of the test set and the size of the training set is $40 \%$.

Once several training sets have been created, the same architecture is trained several times starting from a random initial condition and the performance on the complementary set is evaluated. Once several models have been obtained, the model with lowest test error is selected. The selected model is then tested on a different dataset to verify the generalization capabilities of the obtained estimator. This procedure allows to fully exploit a dataset with 2878444 entries.

\section{NUMERICAL RESULTS}

Previous section provided a description of the method. This section shows some preliminary results obtained with the MBCV method using the dataset provided by Piaggio Aerospace. To fully understand the possibilities given by this method, both the training set and the estimation error have been analysed. Figure 3 allows to compare the size of the training sets obtained with the application of the proposed

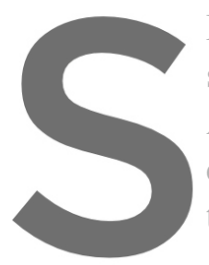
MBCV method. As it can be seen from Figure 3, the size of the test
size of the training set and this is an important figure on the generaliz
Although the method tends to use always the higher number of points
chances to overfit. In fact, the size of the test set is considered non-1
the training set.

A demonstration that a different combination of flight segments can actually lead to a better estimator

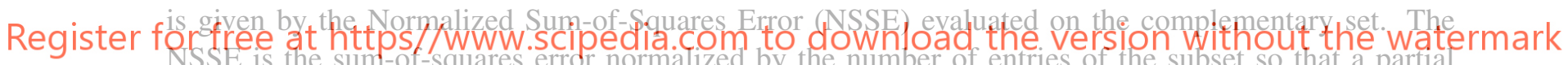
compensation for the size is provided. Figure 4 collects the NSSE values on the selected CV test sets providing a strong evidence that a different selection of the training set leads to a lower test error. The average value is also reported as a red line as important figure for the model average methods.

Figure 5 collects the error normalized by the size of the set obtained after every training operation on a logarithmic chart. A clear difference can be observed between the error obtained on the training set with respect to the error obtained on the test set and this is a known phenomena when data comes from flight simulators. Even when sensor noise and measurement delay are implemented, the training error can be reduced to very low values when data is generated by a mathematical model. This should not be confused with overfitting, because the test error does not show an increase during the training phase.

The analysis of the timeseries is also of great importance because it gives a quick demonstration of the estimator capabilites. Figure 6 shows two examples of partitions used as training set on the selected estimator. The first one is related to a steady state condition whereas the second one contains a 3-2-11 elevator command. As it can be seen from Figure 6, in both case the estimation error of the initial estimation is non-negligible. This could be one of the reasons why these two segments resulted to be 

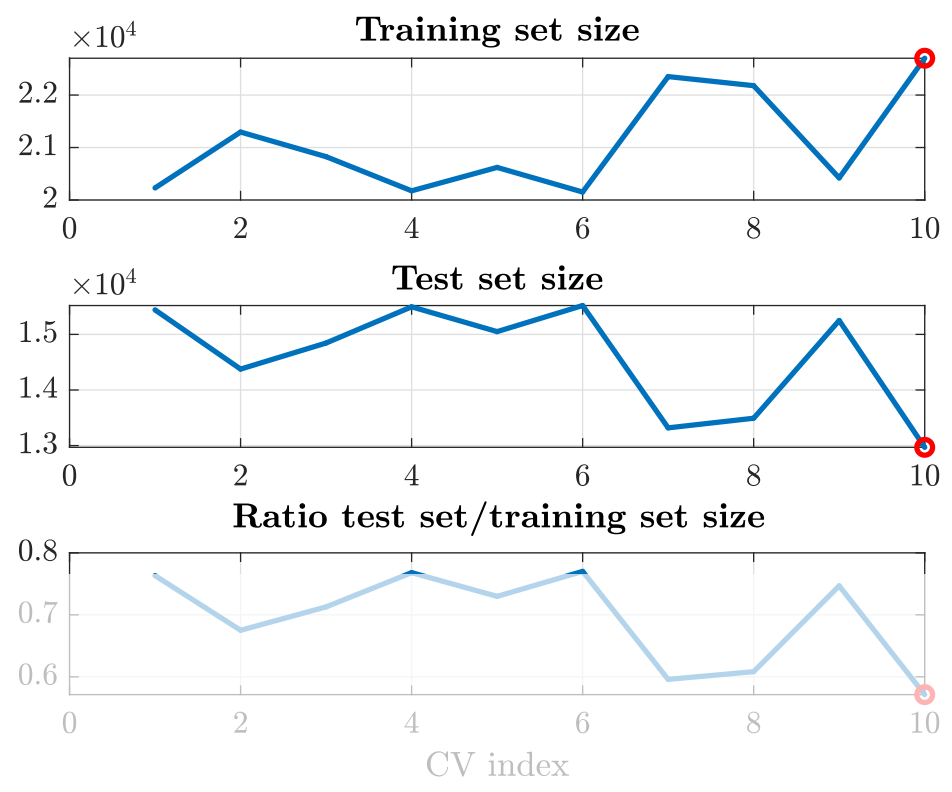

Figure 3: Comparison of the size of the training and test sets for the various MBCV partitions.
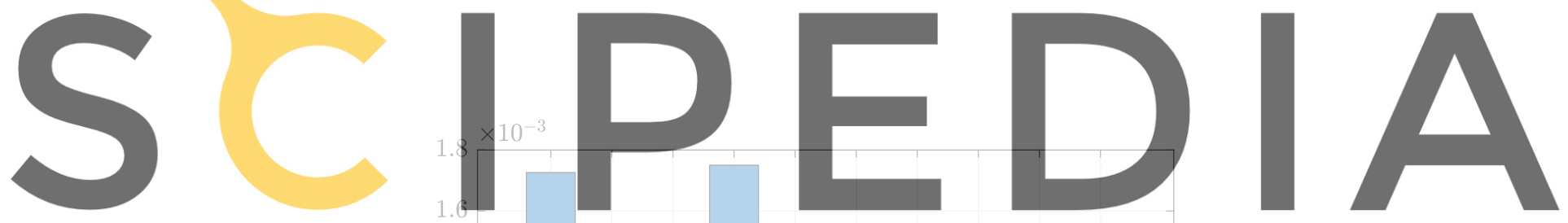

Register for free at https//wwww.scipedia.com to download the version without the watermark

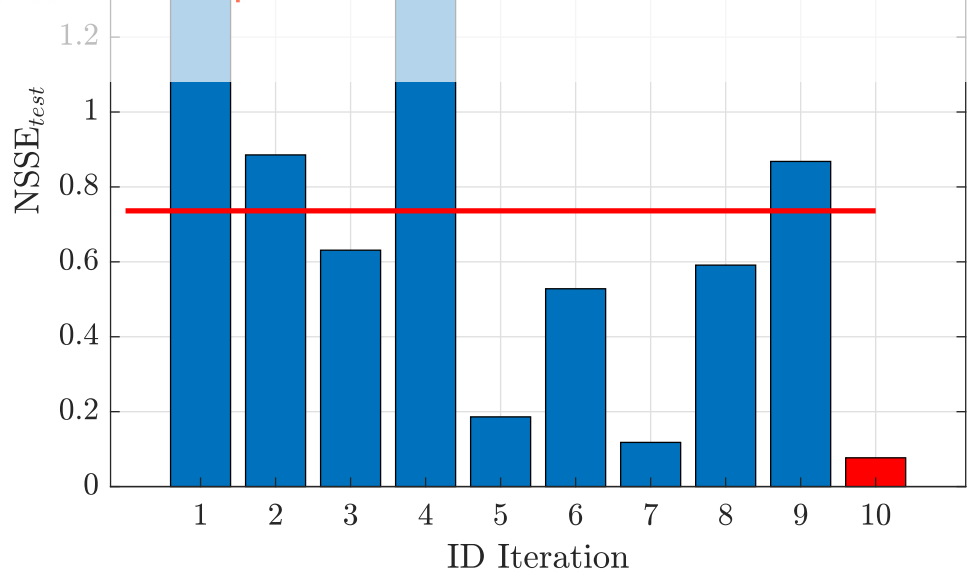

Figure 4: Comparison of the NSSE evaluated on the various test sets. The red line represents the average value 


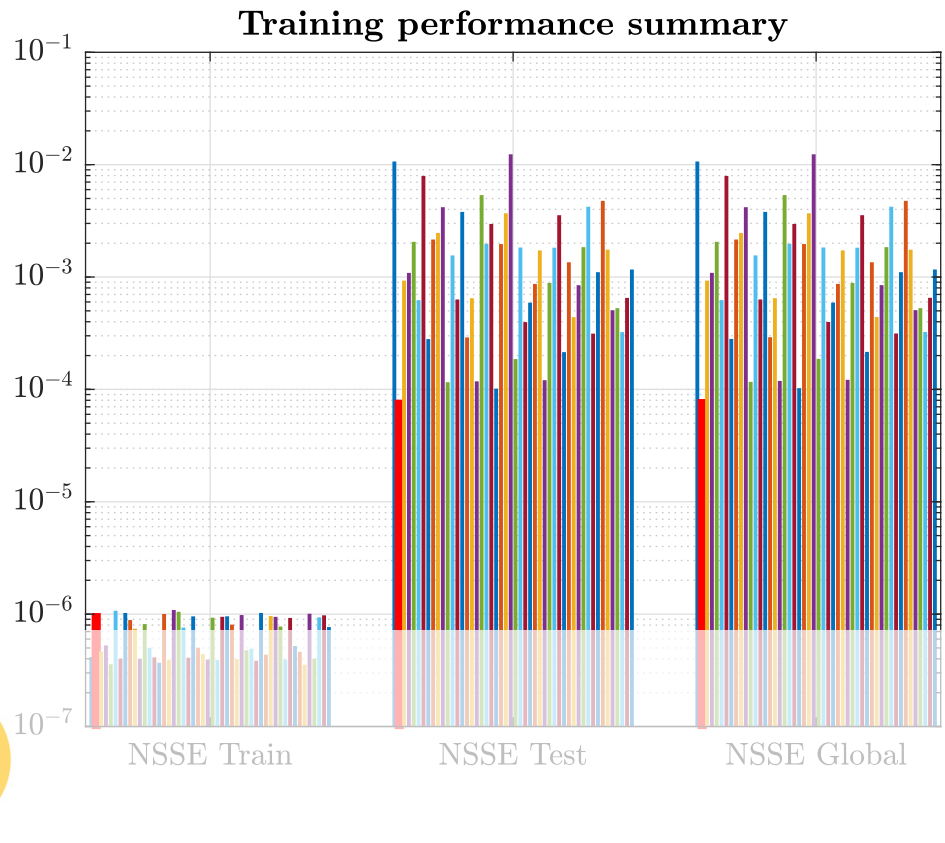

Figure 5: Error obtained after every training operation on a logarithmic chart. The highlighted bar represents the selected estimator.

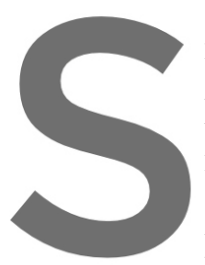

informative in the $\mathrm{MBC}$

Figure 7 shows the time history

noeuver, which is a partic

A first analysis of uncert

Density Fun

reported in Figure 8. The reduction of accuracy on the test set is expected, however the result is still

Register forcefreaslat https//www.scipedia.com to download the version without the watermark

\section{CONCLUSIONS}

Synthetic sensors based on NN for the estimation of AOA/AOS are deeply affected by the selection of the training dataset. At the time of writing this paper, a guideline of which manoeuvers result in the lowest estimation error in this application is still missing. This work gives evidence that a careful definition of the training set can help to reduce the error peaks without modifying the neural architecture, provided a sufficient number of neurons. The proposed method, called MBCV, is a CV procedure applied after a partitioning based on the flight manoeuvers. The NSSE evaluated on the test set resulted to be reduced of about 2 orders of magnitude on the selected model. Unfortunately, it was not possible to define a best set of manoeuvers, but the clear effect of the different composition of training sets gives evidence that a solution might be found in the future. A drawback of this method is that the computational cost increases with the number of possible training sets and, sometimes, the various partitions contain more than a singular flight maneuver in order to reduce the number of trials. A further study will be conducted on the CV scheme to optimize the ratio between the size of the test set and the size of the training set. 

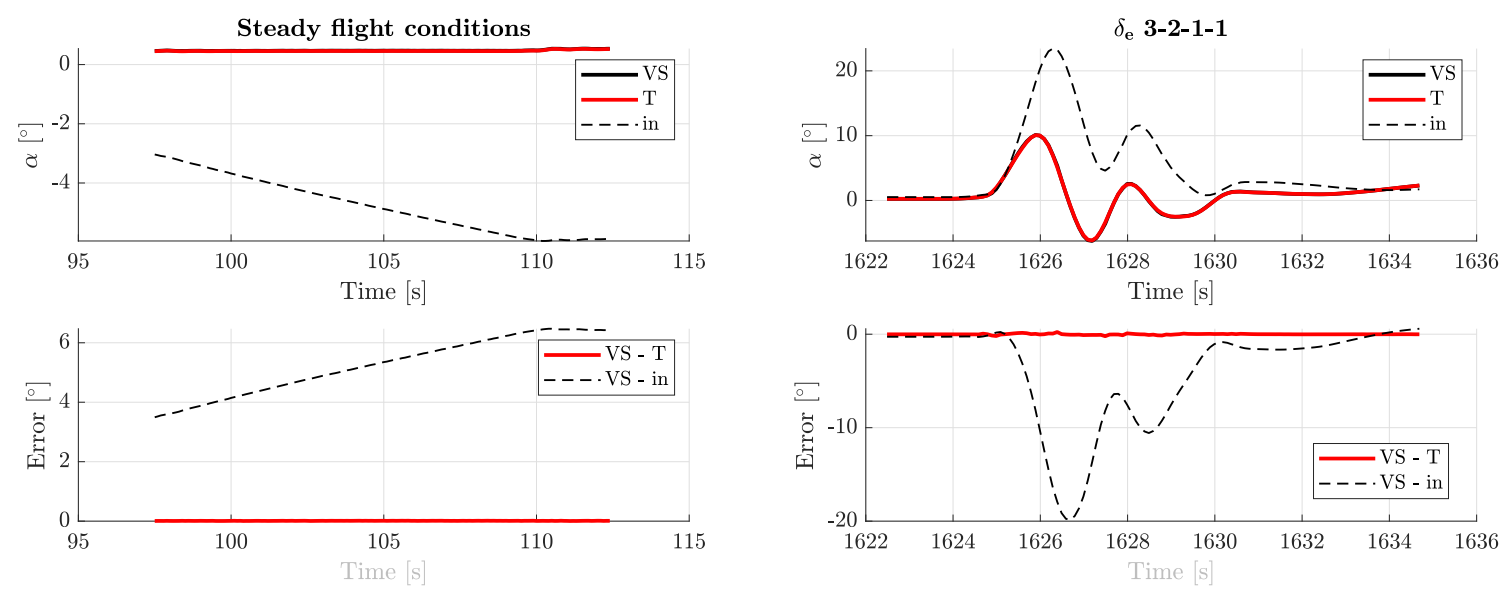

Figure 6: Time history of two segments of the final training set of the AOA signal in steady state condition and during a 3-2-1-1 elevator command (VS: virtual sensor, T: true value, in: initial estimation)
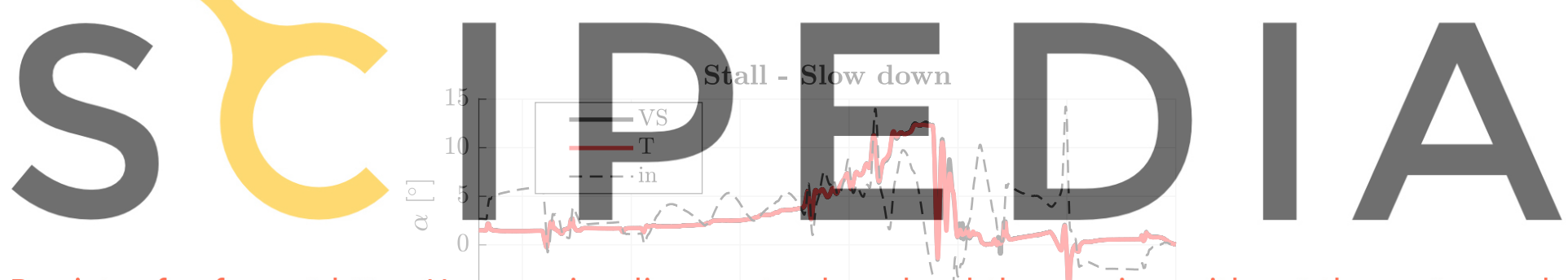

Register for free at https//Wwww.scipedia.com to downloald the version without the watermark $\begin{array}{rr}350 \quad 400 \quad 500 \\ & \text { Time }[\mathrm{s}]\end{array}$

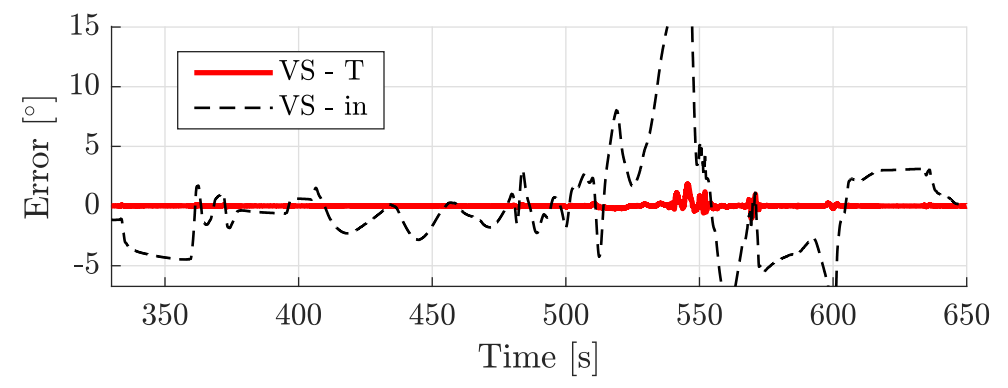

Figure 7: Time history of the AOA signal during a stall manoeuver for the generalization test (VS: virtual sensor, $\mathrm{T}$ : true value, in: initial estimation) 


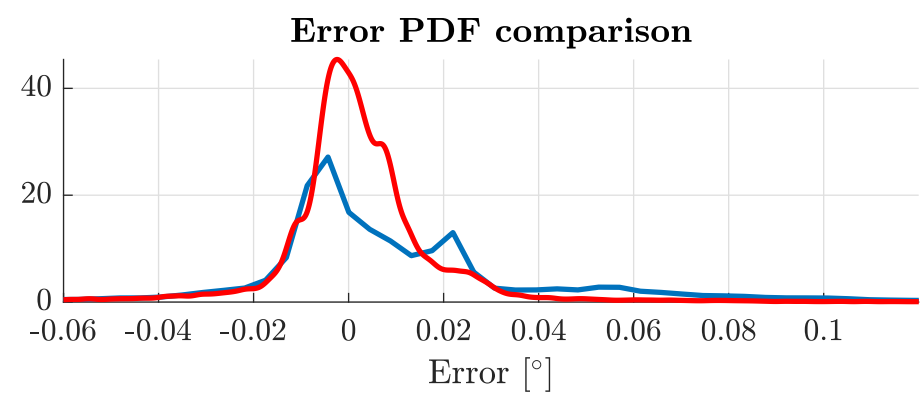

Error CDF comparison

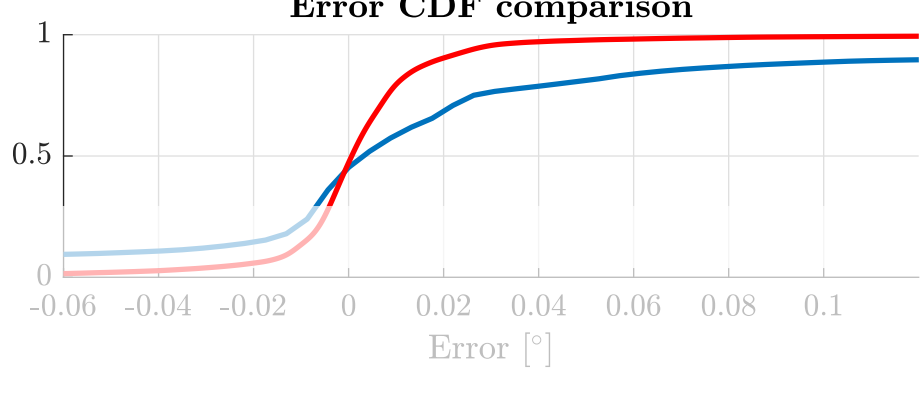

Figure 8: Error PDF and CDF on the training (red) and generalization test set (blue).

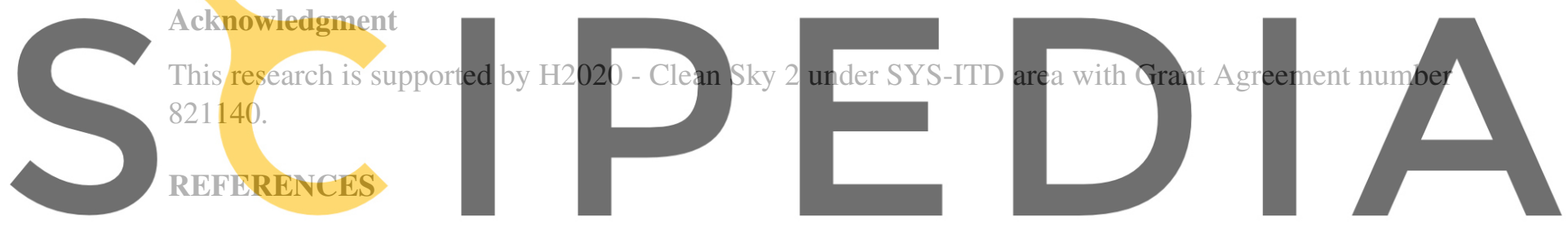

[1] Sankaralingam, L. and Ramprasadh, C., "A comprehensive survey on the methods of angle of attack

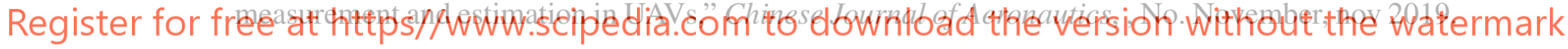

[2] Wise, K. A., "Computational Air Data System for Angle-Of-Attack and Angle-Of-Sideslip," 2005, US 6,928,341 B2.

[3] Prem, S., Sankaralingam, L., and Ramprasadh, C., "Pseudomeasurement-Aided Estimation of Angle of Attack in Mini Unmanned Aerial Vehicle," Journal of Aerospace Information Systems, Vol. 17, No. 11, 2020, pp. 603-614.

[4] Colgren, R., The feasibility of using an INS for control system feedbacks.

[5] Colgren, R. D., "Method and System for Elimination and Correction of Angle of Attack and Sideslip Angle from Acceleration Measurements," 2001, Patent No. 6,273,370 B1.

[6] Colgren, R., Frye, M., and Olson, W., "A proposed system architecture for estimation of angleof-attack and sideslip angle," Guidance, Navigation, and Control Conference and Exhibit, No. c, American Institute of Aeronautics and Astronautics, Reston, Virigina, aug 1999, pp. 743-750.

[7] Colgren, R. and Martin, K., "Flight test validation of sideslip estimation using inertial accelerations," AIAA Guidance, Navigation, and Control Conference and Exhibit, No. August, American 
Institute of Aeronautics and Astronautics, Reston, Virigina, aug 2000.

[8] Li, R., Lu, C., Liu, J., and Lei, T., "Air Data Estimation Algorithm under Unknown Wind Based on Information Fusion," Journal of Aerospace Engineering, Vol. 31, No. 5, sep 2018, pp. 04018072.

[9] Rohloff, T. J., Whitmore, S. A., and Catton, I., "Air data sensing from surface pressure measurements using a neural network method,” AIAA Journal, Vol. 36, No. 11, 1998, pp. 2094-2101.

[10] Samara, P. A., Fouskitakis, G. N., Sakellariou, J. S., and Fassois, S., "Aircraft angle-of-attack virtual sensor design via a functional pooling narx methodology," 2003 European Control Conference (ECC), IEEE, sep 2003, pp. 1816-1821.

[11] Lerro, A., Battipede, M., Gili, P., and Brandl, A., "Aerodynamic angle estimation: comparison between numerical results and operative environment data," CEAS Aeronautical Journal, Sep 2019.

[12] Lerro, A., Brandl, A., and Gili, P., "Model-Free Scheme for Angle-of-Attack and Angle-of-Sideslip Estimation," Journal of Guidance, Control, and Dynamics, Vol. 0, No. 0, 12 2020, pp. 1-6.

[13] Langelaan, J. W., Alley, N., and Neidhoefer, J., "Wind Field Estimation for Small Unmanned Aerial Vehicles," Journal of Guidance, Control, and Dynamics, Vol. 34, No. 4, jul 2011, pp. 1016-1030.

[14] Lerro, A., Battipede, M., Gili, P., Ferlauto, M., Brandl, A., Merlone, A., Musacchio, C., Sangaletti, G., and Russo, G., "The Clean Sky 2 MIDAS Project - an Innovative Modular, Digital and Integrated Air Data System for Fly-by-Wire Applications," 2019 IEEE 5th International Workshop on Metrology for AeroSpace (MetroAeroSpace), June 2019, pp. 714-719.

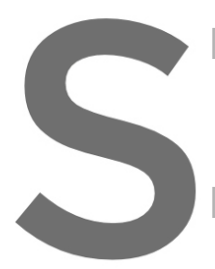

[15] Lerro, A., Brandl, A Sensor for Aerodyn 2019

[16] Brandl, A. and Battipede, Aerodynamic Angles A., Battipede, M., and Gi
amic Angle Estimation
tipede, M. "Effects of the
es of an Acrial Vehicle,"
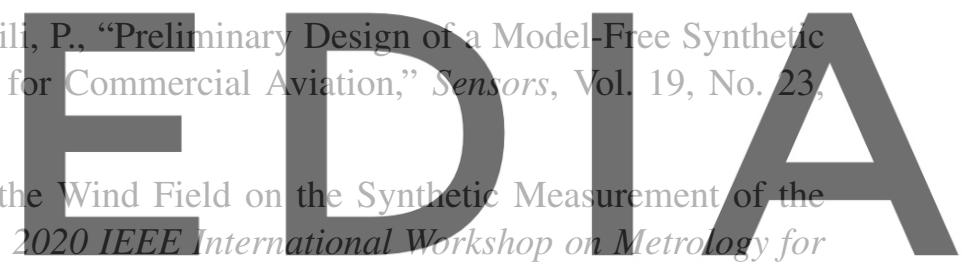
AeroSpace, 6 2020, pp. 185-190.

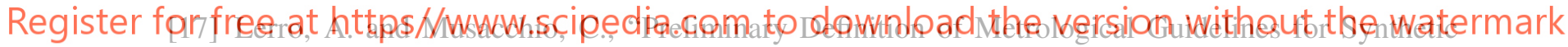
Sensor Verification," 2020 IEEE International Workshop on Metrology for AeroSpace, 6 2020, pp. $191-196$.

[18] Lerro, A., Brandl, A., Battipede, M., and Gili, P., "A Data-Driven Approach to Identify Flight Test Data Suitable to Design Angle of Attack Synthetic Sensor for Flight Control Systems," Aerospace, Vol. 7, No. 5, may 2020, pp. 63.

[19] Lerro, A. and Musacchio, C., "Preliminary Definition of Metrological Guidelines for Synthetic Sensor Verification," 2020 IEEE 7th International Workshop on Metrology for AeroSpace (MetroAeroSpace), 2020, pp. 187-192.

[20] Arlot, S. and Celisse, A., "A survey of cross-validation procedures for model selection," Statist. Surv., Vol. 4, 2010, pp. 40-79. 